\title{
The incidence and predictors of symptomatic venous thromboembolism associated with peripherally inserted central catheters in patients with nasopharyngeal carcinoma
}

This article was published in the following Dove Press journal: OncoTargets and Therapy

\author{
Yu-Jing Liang ${ }^{1,2, *}$ \\ Yan $\mathrm{He}^{1, *}$ \\ Jian-Mei Li ${ }^{1, *}$ \\ Lin-Min Chen \\ Li-Ping Chen' \\ Cong Wang' \\ Lu Ji' \\ Zhen-Xiu Li' \\ Lin-Quan Tang ${ }^{1,2}$ \\ Qiu-Yan Chen ${ }^{1,2}$ \\ Yu-Ying Fan' \\ Wen $\mathrm{Hu}^{\prime}$
}

'Department of Nasopharyngeal Carcinoma, Sun Yat-Sen University Cancer Center, Guangzhou, China;

${ }^{2}$ State Key Laboratory of Oncology in South China, Collaborative Innovation Center for Cancer Medicine, Sun Yat-Sen University Cancer Center, Guangzhou, China

*These authors contributed equally to this work

Correspondence: Yu-Ying Fan; Wen Hu Department of Nasopharyngeal Carcinoma, Sun Yat-Sen University Cancer Center, 65I Dongfeng East Road, Yuexiu District, Guangzhou 510060,

Guangdong Province, China

Tel +862087343380

Fax +86 2087343392

Email fanyy@sysucc.org.cn;

huwen@sysucc.org.cn
Background: Despite wide usage, peripherally inserted central catheter (PICC)-related venous thromboembolism (VTE) is common in nasopharyngeal carcinoma (NPC) patients.

Patients and methods: This was a retrospective cohort study of NPC patients with PICC insertions from February 2, 2007 to December 25, 2014 in Sun Yat-Sen University Cancer Centre. Univariable and multivariable logistic regression analyses were used to estimate odds ratios (ORs) with 95\% confidence intervals (CIs) for the correlations between risk factors and symptomatic PICC-VTE.

Results: Of the 1,363 NPC patients, 76 developed symptomatic VTE. In univariable analysis, body mass index (BMI), Eastern Cooperative Oncology Group (ECOG) score, metastasis stage (M stage), and VTE history were associated with symptomatic PICC-VTE. Following multivariable adjustments, BMI (OR 0.900, $p=0.007$ ), ECOG score (OR 4.162, $p=0.011$ ), $\mathrm{M}$ stage (OR 2.717, $p=0.019$ ), and VTE history (OR 109.772, $p<0.001$ ) were still statistically significant.

Conclusion: PICC-VTE is a common complication in NPC patients, with an incidence of $5.6 \%$ in our analysis. Those with VTE history and lower BMI and worse ECOG performance score metastatic NPC patients are more susceptible to symptomatic PICC-related thrombosis and thus may require prophylactic anticoagulation.

Keywords: symptomatic venous thromboembolism, peripherally inserted central catheters, nasopharyngeal carcinoma

\section{Background}

Nasopharyngeal carcinoma (NPC) is a malignant tumor prevalent in Southeast Asia, Micronesia/Polynesia, East Asia, and North Africa. A total of 86,700 cases were reported worldwide in 2012, causing 51,000 deaths. ${ }^{1,2}$ The fundamental treatment modality is radiotherapy. According to the National Comprehensive Cancer Network (NCCN) guidelines, concurrent chemotherapy is recommended for locoregionally advanced NPC, and 2-3 cycles of chemotherapy with single-agent cisplatin regimen are frequently used. Besides, since nephrotoxicity is a major side effect of cisplatin, adequate hydration is needed to prevent renal damage. Therefore, intravenous infusion for NPC patients lasts for a long time and spans over time. Furthermore, during radiotherapy, parenteral nutrition support is required for the difficulty in feeding due to pain caused by radioactive mucositis. In conclusion, in this modern era, peripherally inserted central catheters (PICCs) are almost universally used in NPC patients. 
The use of PICC makes parenteral nutrition, blood drawing, and other intravenous treatments more convenient. ${ }^{3,4}$ In addition, with the development of the vascular access nursing teams, the success rate of this nurse-operated central venous catheterization is increasing, and the maintenance experience is getting richer. ${ }^{5}$ PICC has become more accessible to chemotherapy in NPC patients, which reduces the pain of frequent puncture and meets the demands for intermittent treatment.

Despite the above advantages, PICC conveys a higher risk of catheter-related venous thromboembolism (VTE), which is a common and potentially dangerous complication. ${ }^{6-8}$ Although many cases of thrombosis induced by PICC are asymptomatic, it can cause recurrence, postthrombotic syndrome, and the most severe form of adverse event, pulmonary embolism. ${ }^{4,9}$ There is a strong correlation between PICC and VTE, especially in critically ill patients or patients with malignant tumors. ${ }^{10}$ Research studies have reported that PICC gauge, catheter material, infection, previous catheterization, metastatic, and other factors may increase the risk of VTE associated with PICCs. ${ }^{4,5}$ Studies have also suggested that infection, positive family history of thrombosis, diabetes, advanced disease, and chronic obstructive pulmonary disease (COPD) are associated with developing PICC-related VTE in chemotherapy patients. ${ }^{11,12}$ However, data of the incidence and predictors of thrombosis in NPC patients with or without chemotherapy or radiotherapy are poorly documented. Therefore, we carried out a retrospective cohort study to identify the incidence and predictors of symptomatic VTE associated with PICC in patients with NPC.

\section{Patients and methods}

A retrospective cohort study of NPC patients who underwent PICC insertions from February 2, 2007 to December 25, 2014 was conducted in the Sun Yat-Sen University Cancer Centre. Before treatment, the patients experienced a series of inspections including head and neck examination, nasopharyngoscopy and biopsy, magnetic resonance imaging scans of head and neck, chest computed tomography or radiography, abdominal ultrasonography, positron emission tomography/ computed tomography, conventional electrocardiograph, and a series of blood sampling like blood routine, biochemical routine, coagulation function, and Epstein-Barr virus (EBV) DNA. Patients were enrolled if they 1) had been diagnosed with NPC and 2) had PICC placement. Basic information and clinical data on potential risk factors for thrombosis were documented. Patients whose clinical or device data were missing were excluded.

\section{PICC insertion and maintenance}

Before insertions, PICC-relevant education was provided for patients, and informed consents were signed. All PICC catheterizations were operated normatively by our extensively trained vascular access nursing team with the guidance of ultrasound. To encourage patients to exercise more, the preferred puncture site was the right arm. And if it is inappropriate, the left arm would be the substitution. Before insertion, we measure the length of the catheter and the arm circumference $10 \mathrm{~cm}$ above the fossa cubitalia. Basilic vein was the first choice for puncture. When the basilic vein was unsuitable or previously thrombosed, we use the cephalic or brachial veins as alternatives. The tip locations were determined by conventional chest radiographs. If the tip location of PICC was not correct, the tip was repositioned by a senior nurse or the patient was transferred to the radiology department and the tip was repositioned by an experienced interventional radiologist under fluoroscopy.

Nurses of our vascular access nursing team provided maintenance care for all PICC weekly or earlier when necessary. During maintenance, whether the puncture site was normal, circumferences of the upper arms (be aware of $>3 \mathrm{~cm}$ ), whether the catheter was leaking or blocking, and other abnormalities were observed. All catheters were flushed regularly with saline and locked by using $0-100 \mathrm{IU} / \mathrm{mL}$ heparin saline; the dressing was changed within 24 hours after catheter placement. Transparent dressings were changed every 5-7 days while gauze dressings were changed every other day, or at any time if dressings became wet, dirty, or loose. PICCs were removed in either of the following cases: no complication associated with PICC at the end of treatment, death of patients, giving up treatments, or requirements for removal.

\section{Variables and definitions}

It has been reported that the incidence of thrombosis associated with PICC in chemotherapy patients is associated with patient-, provider-, and device-related characteristics. General items include insertion adjustments, type of PICC, number of lumens, PICC gauge, stage of carcinoma, coagulation function, and others. ${ }^{5,11,13}$ In addition, NPC is highly associated with EBV infection. ${ }^{14,15}$ Hence, in NPC patients, we were specifically interested in whether there is a connection between EBV infection, the amount of virus, and VTE. Moreover, we also paid attention to the influence of treatment modality on VTE. Therefore, risk factors of symptomatic thrombosis associated with PICC in patients with NPC were divided into four aspects to explore in this 
article, summarizing as patient-, treatment-, provider-, and device-related characteristics (Figure 1).

All therapy regimens were made according to the recommendations of NCCN guidelines and patients' own situation. VTE was diagnosed by color Doppler ultrasound, mainly manifesting as incompressibility of the vein with or without visible intraluminal thrombus. Of all the patients, screening was performed only when symptoms suggested suspicious thrombosis, such as arm edema, swelling and pain, or chest pain. Once the patient was suspected to develop VTE, blood coagulation function was tested at the same time. Routine testing for symptomatic VTE associated with PICC was not performed. The time of thrombosis was defined as the day when thrombosis was diagnosed by ultrasound.

Diagnosed as NPC, patients were staged according to the 7th edition UICC (Union for International Cancer Control) staging system. Common comorbidities include hypertension, diabetes mellitus, coronary artery disease, and hepatitis B. The body mass index (BMI), a value generally used to assess the nutritional status, is defined as the body mass divided by the square of the body height. The normal BMI for Asian adults is $18.5-23.0 \mathrm{~kg} / \mathrm{m}^{2}$. The Eastern Cooperative Oncology Group (ECOG) performance status describes a patient's level of functioning in terms of their ability to care for themselves including activities of daily living, and the ECOG performance score were used to assess how the disease affects the patients' daily life by doctors. The cutoff value of ECOG to distinguish fully normal and restricted is 1. The distinction between high and low EBV DNA levels is 4,000 copies $/ \mathrm{mL}$.

\section{Statistical analysis}

The baseline characteristics of this cohort was characterized by descriptive statistics. The proportion of patients with a symptomatic PICC-VTE was reported. Continuous variables of the asymmetric distribution were reported as median

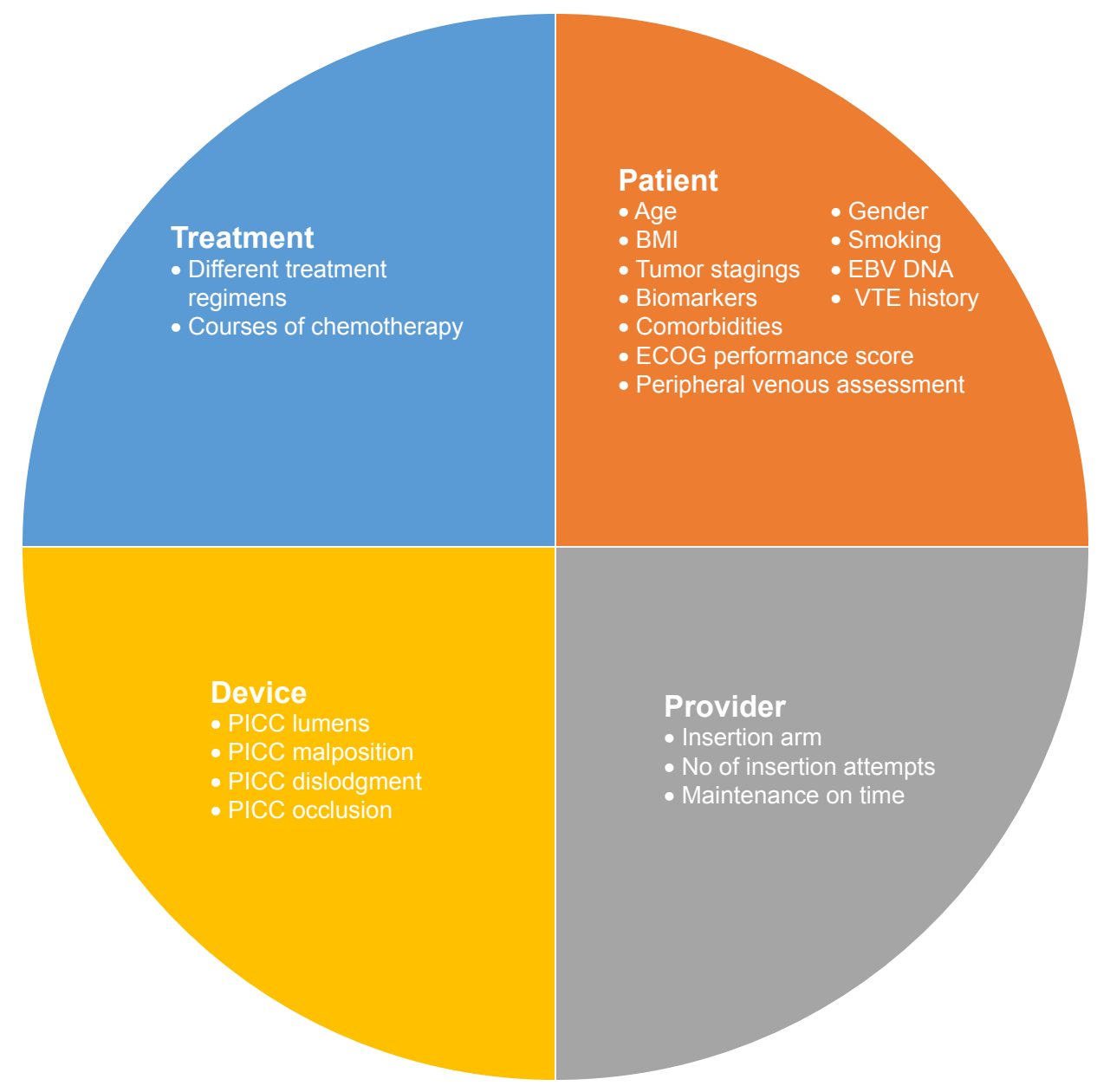

Figure I Conceptual model for PICC-related VTE in NPC patients.

Abbreviations: PICC, peripherally inserted central catheters; VTE, venous thromboembolism; NPC, nasopharyngeal carcinoma; BMI, body mass index; ECOG, Eastern Cooperative Oncology Group; EBV, Epstein-Barr virus. 
(interquartile range), while symmetric distribution data were reported as mean, and categorical variable were presented as the number of cases (\%). Univariable and multivariable logistic regression analyses were used to estimate the unadjusted and adjusted odds ratios (ORs) and 95\% confidence intervals (CIs) for the correlations between risk factors and symptomatic PICC-VTE. Statistical analyses were conducted with the SPSS 24.0 (IBM Corporation, Armonk, NY, USA) software. All statistical tests were two-tailed; $p<0.05$ was considered to indicate statistical significance. The study was approved by the ethics committee of the Sun Yat-Sen University Cancer Center.

\section{Results}

Between February 2, 2007 and December 25, 2014, 1,703 PICC were inserted in patients with NPC in Sun Yat-Sen University Cancer Centre. Six patients without a recorded removal date were excluded; 36 patients' height, weight, and other basic information was lost, and 298 had no documented coagulation test results (Figure 2). Therefore, a total of 1,363 patients were finally included in our study. There were 1,004 (73.66\%) men and 259 women $(26.34 \%)$, with a mean age of 44.71 years (Table 1 ).

Until catheters were removed, 76 patients developed symptomatic PICC-VTE, accounting for 5.6\% among the 1,363 NPC patients with PICC insertion. Of these 76 patients, seven had an metastasis stage (M stage), taking up 9.21\%. There were 42 patients with right-hand catheterization, accounting for $55.26 \%$. Figure 3 shows the cumulative incidence of symptomatic PICC-VTE. With respect to veins, a majority of thrombosis were developed in more than one vein. The most common PICC-VTE involved basilic vein and subclavian vein, accounting for $71.05 \%$ cases (54) and $39.47 \%$ cases (30) and that involving axillary vein and cephalic vein were $31.58 \%$ (24) and $10.53 \%$ (8) while the least were brachial vein (4), ulnar vein (2), and median vein (1). The median follow-up time was 27.80 months until January 1 , 2015. During follow-up, neither the 76 patients developed VTE recurrence, postthrombotic syndrome, or pulmonary embolism nor new cases were reported.

In univariable analysis, a large number of relevant data including patient-, treatment-, provider-, and devicerelated characteristics were analyzed. We find that BMI (OR 0.898, $p=0.004$ ), ECOG performance score (OR 4.637, $p=0.005$ ), M stage (OR 2.459, $p=0.033$ ), and VTE history (OR 90.563, $p \leq 0.001$ ) were associated with symptomatic PICC-VTE in patients with NPC. The association between symptomatic PICC-VTE and other factors such as gender, age, comorbidities, PICC lumens, and insertion arms was not found (Table 2). After multivariable adjustments, BMI (OR 0.900, $p=0.007$ ), ECOG score (OR 4.162, $p=0.011)$, M stage (OR 2.717, $p=0.019$ ), and VTE history

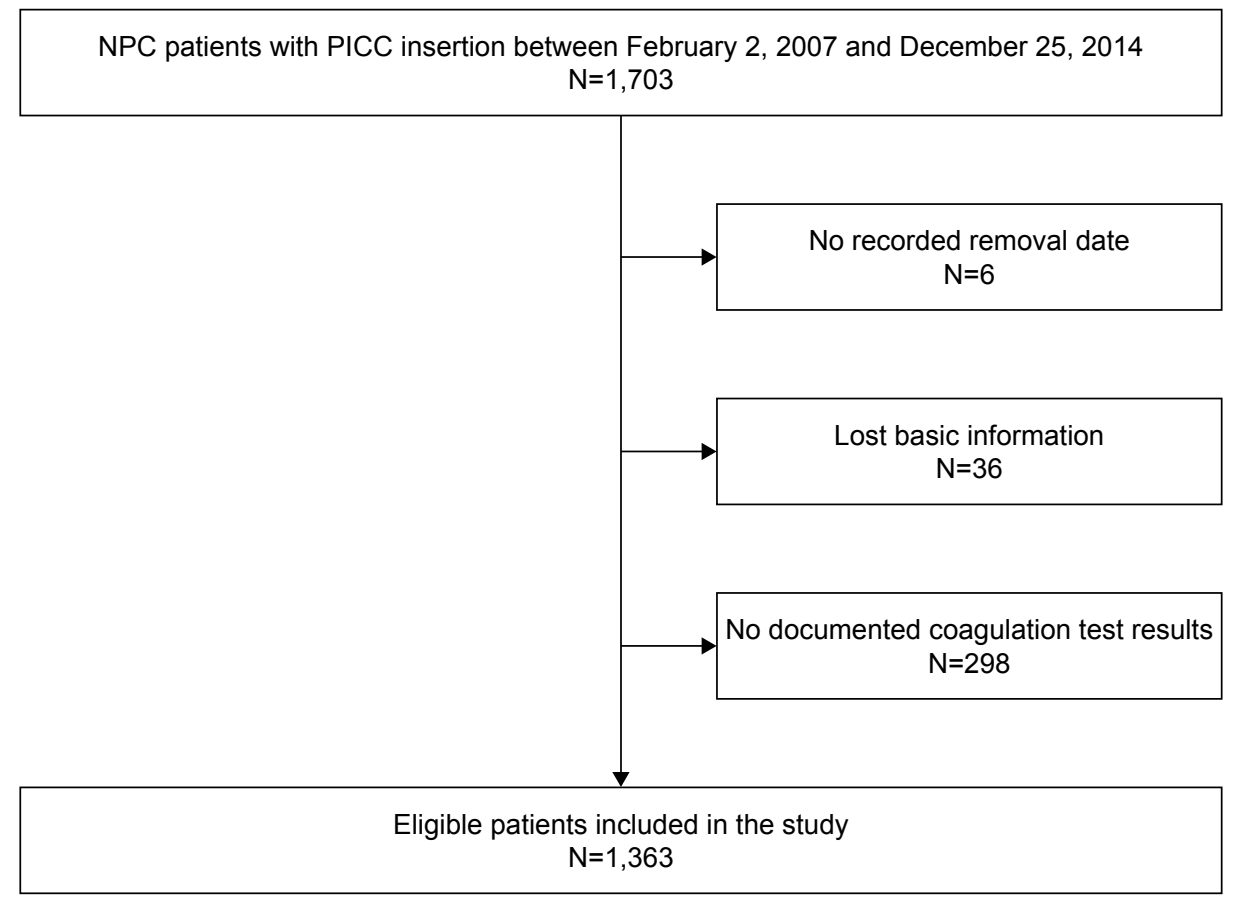

Figure 2 Flowchart to describe the generation of final study cohort.

Abbreviations: PICC, peripherally inserted central catheters; NPC, nasopharyngeal carcinoma. 
Table I Characteristics of NPC patients with PICC insertion

\begin{tabular}{|c|c|c|}
\hline Variables & $\begin{array}{l}\text { PICC-VTE } \\
(\mathrm{N}=76)\end{array}$ & $\begin{array}{l}\text { No PICC-VTE } \\
(\mathrm{N}=\mathrm{I}, 287)\end{array}$ \\
\hline Age (years) & $46.33 \pm 11.77$ & $44.62 \pm 11.03$ \\
\hline Male gender, n (\%) & $54(71.00)$ & $950(73.82)$ \\
\hline BMI $\left(\mathrm{kg} / \mathrm{m}^{2}\right)$ & $21.96 \pm 3.23$ & $23.09 \pm 3.32$ \\
\hline Smoking, n (\%) & $23(30.26)$ & $424(32.94)$ \\
\hline $\mathrm{HGB}(\mathrm{g} / \mathrm{L})$ & $140.85 \pm 16.67$ & $|42.7| \pm \mid 4.50$ \\
\hline PLT (109/L) & $238.50 \pm 72.87$ & $240.45 \pm 62.35$ \\
\hline PT $(s)$ & $12.03 \pm 4.23$ & $11.19 \pm 0.84$ \\
\hline PT\% (\%) & $96.39 \pm 21.81$ & $103.13 \pm 17.47$ \\
\hline INR & $1.05 \pm 0.32$ & $0.99 \pm 0.07$ \\
\hline APTT (s) & $26.48 \pm 5.68$ & $26.12 \pm 3.62$ \\
\hline $\mathrm{DD}(\mu \mathrm{g} / \mathrm{mL})$ & $0.30(0.43)$ & $0.31(0.43)$ \\
\hline ECOG score & $1.05 \pm 0.23$ & $1.00 \pm 0.15$ \\
\hline \multicolumn{3}{|l|}{ Comorbidities, n (\%) } \\
\hline Hypertension & 6 (7.89) & $89(6.91)$ \\
\hline DM & $2(2.63)$ & $39(3.03)$ \\
\hline НерB & $9(11.84)$ & $108(8.39)$ \\
\hline VTE history, n (\%) & $6(7.89)$ & I $(0.08)$ \\
\hline Peripheral venous assessment & $1.39 \pm 0.75$ & $1.35 \pm 0.65$ \\
\hline \multicolumn{3}{|l|}{ PICC lumens, n (\%) } \\
\hline I & $68(89.47)$ & I, $197(93.00)$ \\
\hline 2 & $8(10.53)$ & $90(7.00)$ \\
\hline PICC malposition, n (\%) & $8(10.53)$ & $73(5.67)$ \\
\hline PICC dislodgment, n (\%) & $6(7.89)$ & $40(3.11)$ \\
\hline PICC occlusion, n (\%) & $5(6.58)$ & $58(4.5 \mathrm{I})$ \\
\hline \multicolumn{3}{|l|}{ Insertion arm, n (\%) } \\
\hline Left & $34(44.74)$ & $412(32.01)$ \\
\hline Right & $42(55.26)$ & $875(67.99)$ \\
\hline No of insertion attempts & $1.03 \pm 0.23$ & $1.02 \pm 0.20$ \\
\hline Maintenance on time, $\mathrm{n}(\%)$ & $70(92.11)$ & I, 197 (93.0I) \\
\hline \multicolumn{3}{|l|}{ T stage, $n(\%)$} \\
\hline 1 & $24(31.58)$ & $48(3.73)$ \\
\hline 2 & $39(5 I .3 I)$ & $210(16.32)$ \\
\hline 3 & $10(13.16)$ & $679(52.76)$ \\
\hline 4 & $3(3.95)$ & $350(27.20)$ \\
\hline \multicolumn{3}{|l|}{ N stage, n (\%) } \\
\hline 0 & $8(10.53)$ & 135 (10.49) \\
\hline 1 & $28(36.84)$ & $448(34.8 I)$ \\
\hline 2 & $32(42.10)$ & $520(40.40)$ \\
\hline 3 & $8(10.53)$ & $184(14.30)$ \\
\hline \multicolumn{3}{|l|}{ M stage, n (\%) } \\
\hline 0 & 61 (90.79) & I,238 (96.04) \\
\hline 1 & $7(9.21)$ & $51(3.96)$ \\
\hline \multicolumn{3}{|l|}{ UICC stage, n (\%) } \\
\hline 1 & I (I.32) & $5(0.39)$ \\
\hline II & $6(7.89)$ & $86(6.68)$ \\
\hline III & $38(50.00)$ & $693(53.85)$ \\
\hline IV & 31 (40.79) & $503(39.08)$ \\
\hline \multicolumn{3}{|l|}{ EBV DNA (copy/mL) } \\
\hline Low & $38(50.00)$ & $633(49.18)$ \\
\hline High & $38(50.00)$ & $654(50.82)$ \\
\hline \multicolumn{3}{|l|}{ Treatment, n (\%) } \\
\hline $\mathrm{RT}$ & $\mathrm{I}(\mathrm{I} .3 \mathrm{I})$ & $22(\mid .7 I)$ \\
\hline CCRT & $24(31.58)$ & $483(37.53)$ \\
\hline $\mathrm{IC}+\mathrm{RT}$ & $9(1 \mathrm{I} .84)$ & 171 (13.29) \\
\hline $\mathrm{IC}+\mathrm{CCRT}$ & $34(44.74)$ & $572(44.44)$ \\
\hline CT & $8(10.53)$ & $39(3.03)$ \\
\hline
\end{tabular}

Table I (Continued)

\begin{tabular}{|c|c|c|}
\hline Variables & $\begin{array}{l}\text { PICC-VTE } \\
(\mathbf{N}=76)\end{array}$ & $\begin{array}{l}\text { No PICC-VTE } \\
(\mathbf{N}=I, 287)\end{array}$ \\
\hline Courses of chemotherapy & $3.82 \pm 1.88$ & $3.68 \pm 1.71$ \\
\hline \multicolumn{3}{|c|}{$\begin{array}{l}\text { Note: Data shown as mean } \pm \text { standard deviation unless indicated otherwise. } \\
\text { Abbreviations: BMI, body mass index; HGB, hemoglobin; PLT, platelets; PT, } \\
\text { prothrombin time; INR, international normalized ratio; APTT, activated partial } \\
\text { thromboplastin time; DD, D-dimer; ECOG, Eastern Cooperative Oncology Group; } \\
\text { DM, diabetes mellitus; HepB, hepatitis B; VTE, venous thromboembolism; PICC, } \\
\text { peripherally inserted central catheters; RT, radiotherapy; CCRT, concurrent } \\
\text { chemoradiotherapy; IC, induction chemotherapy; CT, chemotherapy; EBV, Epstein- } \\
\text { Barr virus. }\end{array}$} \\
\hline
\end{tabular}

(OR 109.772, $p<0.001$ ) were still statistically significant for predicting symptomatic PICC-VTE in patients with NPC (Table 3).

\section{Discussion}

Our study is the first to analyze the incidence and predictors of symptomatic thrombosis associated with PICC in a large sample of NPC patients. In our retrospective cohort study of consecutive NPC patients with PICC insertions, the incidence of symptomatic VTE was 5.6\%, the result is in accordance with previously published studies, ranging from $0.3 \%$ to $28.3 \%{ }^{16,17}$ Four factors were found to be associated with symptomatic VTE in NPC patients. Among them, three were risk factors, and the remaining one was a protective factor.

The three risk factors that predicted a higher risk of symptomatic VTE in NPC patients with PICC insertions were ECOG score, VTE history, and metastasis stage. We discovered that VTE history was the most significant predictor (OR 109.772, $p<0.001$ ) in our multivariable model, which was consistent with a number of published studies. ${ }^{18,19}$ It was reported that VTE history predicts future episodes of

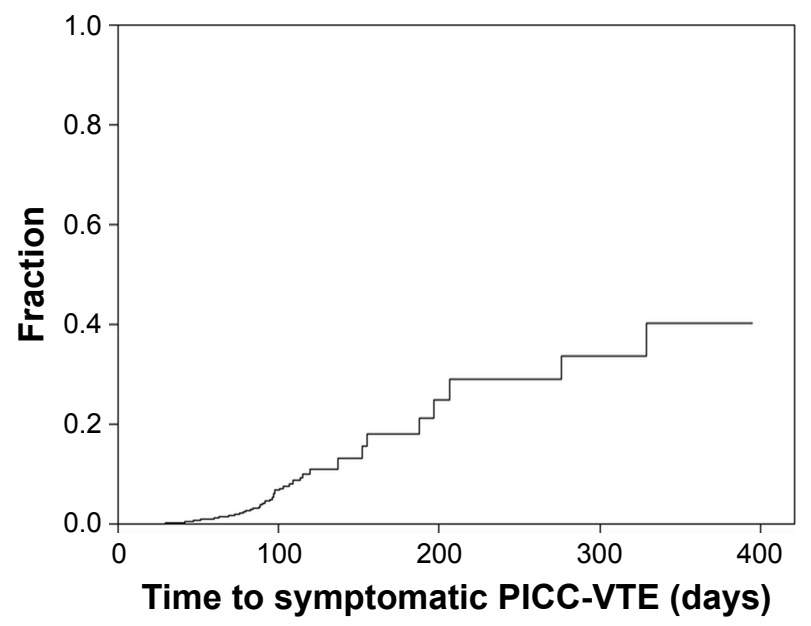

Figure 3 Cumulative incidence of symptomatic PICC-VTE.

Abbreviations: PICC, peripherally inserted central catheters; VTE, venous thromboembolism. 
Table 2 Univariable logistic analysis for risk factors associated with PICC-VTE in NPC patients

\begin{tabular}{|c|c|c|c|}
\hline Variable & OR & $95 \% \mathrm{CI}$ & $p$-value \\
\hline Age (years) (SD) & 1.196 & $0.948-1.509$ & 0.191 \\
\hline \multicolumn{4}{|l|}{ Gender } \\
\hline Female & 1 & Reference & Reference \\
\hline Male & $0.87 I$ & $0.522-1.452$ & 0.595 \\
\hline BMI $\left(\mathrm{kg} / \mathrm{m}^{2}\right)$ & 0.898 & $0.835-0.967$ & 0.004 \\
\hline \multicolumn{4}{|l|}{ Smoking } \\
\hline No & I & Reference & Reference \\
\hline Yes & 0.797 & $0.564-1.128$ & 0.200 \\
\hline HGB (g/L) (SD) & 0.884 & $0.706-1.107$ & 0.282 \\
\hline PLT (10 $\left.10^{\circ} \mathrm{L}\right)(\mathrm{SD})$ & 0.969 & $0.7662-1.225$ & 0.792 \\
\hline PT (s) (SD) & 1.076 & $0.859-1.348$ & 0.522 \\
\hline PT\% (\%) (SD) & 0.800 & $0.631-1.014$ & 0.065 \\
\hline INR & I.199 & $0.05 I-28.28 I$ & 0.910 \\
\hline APTT (s) (SD) & 0.929 & $0.732-1.178$ & 0.543 \\
\hline \multicolumn{4}{|l|}{$\mathrm{DD}(\mu \mathrm{g} / \mathrm{mL})$} \\
\hline$\leq 0.30$ & I & Reference & Reference \\
\hline$>0.30$ & 1.062 & $0.899-1.254$ & 0.482 \\
\hline ECOG score & 4.637 & I.589-13.529 & 0.005 \\
\hline \multicolumn{4}{|l|}{ Hypertension } \\
\hline No & 1 & Reference & Reference \\
\hline Yes & 0.948 & $0.373-2.048$ & 0.910 \\
\hline \multicolumn{4}{|l|}{ DM } \\
\hline No & I & Reference & Reference \\
\hline Yes & 0.856 & $0.205-3.65 I$ & 0.843 \\
\hline \multicolumn{4}{|l|}{ НерВ } \\
\hline No & I & Reference & Reference \\
\hline Yes & 1.466 & $0.712-3.022$ & 0.299 \\
\hline Peripheral venous assessment & 1.097 & $0.784-1.536$ & 0.590 \\
\hline \multicolumn{4}{|l|}{ VTE history } \\
\hline No & I & Reference & Reference \\
\hline Yes & 90.563 & $|0.44|-785.5 \mid 3$ & $<0.001$ \\
\hline \multicolumn{4}{|l|}{ PICC lumens } \\
\hline I & I & Reference & Reference \\
\hline 2 & 1.565 & $0.729-3.357$ & 0.250 \\
\hline \multicolumn{4}{|l|}{ PICC malposition } \\
\hline No & 1 & Reference & Reference \\
\hline Yes & 1.687 & $0.749-3.802$ & 0.207 \\
\hline \multicolumn{4}{|l|}{ PICC dislodgment } \\
\hline No & I & Reference & Reference \\
\hline Yes & 0.855 & $0.377-1.938$ & 0.708 \\
\hline \multicolumn{4}{|l|}{ PICC occlusion } \\
\hline No & I & Reference & Reference \\
\hline Yes & 1.492 & $0.580-3.837$ & 0.406 \\
\hline \multicolumn{4}{|l|}{ Insertion arms } \\
\hline Left & 1 & Reference & Reference \\
\hline Right & 0.855 & $0.527-1.387$ & 0.524 \\
\hline No of insertion attempts & 1.055 & $0.347-3.211$ & 0.925 \\
\hline \multicolumn{4}{|l|}{ Maintenance on time } \\
\hline No & 1 & Reference & Reference \\
\hline Yes & 0.877 & $0.37 I-2.075$ & 0.765 \\
\hline \multicolumn{4}{|l|}{ T stage } \\
\hline 1 & I & Reference & Reference \\
\hline 2 & 0.762 & $0.20 \mathrm{I}-2.874$ & 0.688 \\
\hline 3 & 0.919 & $0.274-3.083$ & 0.891 \\
\hline 4 & 1.097 & $0.318-3.782$ & 0.883 \\
\hline
\end{tabular}

Table 2 (Continued)

\begin{tabular}{|c|c|c|c|}
\hline Variable & OR & $95 \% \mathrm{Cl}$ & $p$-value \\
\hline \multicolumn{4}{|l|}{$\mathrm{N}$ stage } \\
\hline 0 & I & Reference & Reference \\
\hline I & 1.205 & $0.543-2.678$ & 0.647 \\
\hline 2 & 0.909 & $0.405-2.039$ & 0.816 \\
\hline 3 & 0.734 & $0.269-2.004$ & 0.546 \\
\hline \multicolumn{4}{|l|}{ M stage } \\
\hline No & I & Reference & Reference \\
\hline Yes & 2.459 & $1.076-5.618$ & 0.033 \\
\hline \multicolumn{4}{|l|}{ UICC stage } \\
\hline I & I & Reference & Reference \\
\hline II & 0.349 & $0.035-3.483$ & 0.370 \\
\hline III & 0.274 & $0.031-2.405$ & 0.243 \\
\hline IV & 0.308 & $0.035-2.719$ & 0.289 \\
\hline \multicolumn{4}{|l|}{ EBV DNA (copy/mL) } \\
\hline Low & I & Reference & Reference \\
\hline High & 0.964 & I & I \\
\hline \multicolumn{4}{|l|}{ Treatment } \\
\hline RT & I & Reference & Reference \\
\hline CCRT & 1.093 & $0.14 \mid-8.454$ & 0.932 \\
\hline $\mathrm{IC}+\mathrm{RT}$ & 1.158 & $0.140-9.58 \mid$ & 0.892 \\
\hline $\mathrm{IC}+\mathrm{CCRT}$ & $\mathrm{I} .308$ & $0.171-9.993$ & 0.796 \\
\hline $\mathrm{CT}$ & 4.513 & $0.529-38.492$ & 0.168 \\
\hline Courses of chemotherapy & 1.047 & $0.919-1.194$ & 0.491 \\
\hline
\end{tabular}

Note: Data in bold indicates statistical significance $(p<0.05)$. Abbreviations: $\mathrm{OR}$, odds ratio; $\mathrm{Cl}$, confidence interval; $\mathrm{BMI}$, body mass index; HGB, hemoglobin; PLT, platelets; PT, prothrombin time; INR, international normalized ratio; APTT, activated partial thromboplastin time; DD, D-dimer; ECOG, Eastern Cooperative Oncology Group; DM, diabetes mellitus; HepB, hepatitis B; VTE, venous thromboembolism; PICC, peripherally inserted central catheters; RT, radiotherapy; CCRT, concurrent chemoradiotherapy; IC, induction chemotherapy; $\mathrm{CT}$, chemotherapy; EBV, Epstein-Barr virus; $\mathrm{M}$ stage, metastasis stage; $\mathrm{N}$ stage, node stage; UICC, union for international cancer control.

thrombosis; this may indicate that patients are predisposed because of inherited or acquired coagulation disorders. ${ }^{19,20}$ This finding is important because it indicates that NPC patients with VTE may require routine use of anticoagulants after PICC catheterization.

Table 3 Multivariable analysis of risk factors associated with PICC-VTE in NPC patients

\begin{tabular}{llll}
\hline Variable & OR & $\mathbf{9 5 \%} \mathbf{C l}$ & P-value \\
\hline VTE history & & & \\
No & $\mathrm{I}$ & Reference & $\begin{array}{l}\text { Reference } \\
<\mathbf{0 . 0 0 I}\end{array}$ \\
Yes & 109.772 & $12.572-958.468$ & $\mathbf{0 . 0 0 7}$ \\
BMI $\left(\mathrm{kg} / \mathrm{m}^{2}\right)$ & 0.900 & $0.834-0.972$ & $\mathbf{0 . 0 I I}$ \\
ECOG score & 4.162 & $1.385-12.513$ & \\
M stage & & & Reference \\
No & $\mathrm{I}$ & Reference & $\mathbf{0 . 0 1 9}$ \\
Yes & 2.717 & $\mathrm{I} .178-6.266$ & \\
EBV DNA & & & Reference \\
Low & $\mathrm{I}$ & Reference & $0.92 \mathrm{I}$ \\
High & $\mathrm{I} .000$ & $1.000-1.000$ & 0.346 \\
DD $(\mu g / \mathrm{mL})$ & $\mathrm{I} .078$ & $0.922-1.260$ & \\
\hline
\end{tabular}

Note: Data in bold indicates statistical significance $(p<0.05)$.

Abbreviations: OR, odds ratio; $\mathrm{Cl}$, confidence interval; ECOG, Eastern Cooperative Oncology Group; BMI, body mass index; M stage, metastasis stage; VTE, venous thromboembolism; EBV, Epstein-Barr virus; DD, D-dimer; PICC, peripherally inserted central catheters. 
Besides, ECOG performance score was found to be another predictive factor (OR 4.162, $p=0.011)$. In prior published studies, it was reported that poor ECOG performance status or Karnofsky Performance Score (KPS) scale was associated with an increased risk of toxicity caused by chemotherapy. Moreover, impaired functional status is common in old cancer patients or those with multiple metastases. ${ }^{21-23}$ Thus, it may be interpreted that NPC patients with poorer ECOG performance status suffered from more severe toxicities due to radiotherapy with or without chemotherapy and coagulation disorders. An M stage (OR 2.717, $p=0.019$ ) also turned out to be predictive for the risk of thrombosis. VTE is a common complication of the natural history of cancer and its treatment, especially in patients with catheter insertion. ${ }^{24,25}$ Our finding regarding the association between NPC metastasis stage and greater risk of PICC-VTE was in keeping with prior studies. ${ }^{11,26}$ In patients with NPC, the common metastatic sites include bone, liver, lung, or their combinations. Multiple metastatic NPC patients were often confined to bed and thus immobilized. Moreover, NPC patients with metastases are often in a hypercoagulable state. Hence, these clinical conditions usually lead to slower blood flow, resulting in venous stasis, so that these patients were susceptible to VTE. ${ }^{27}$

The only protective factor discovered in our study was BMI (OR 0.900, $p=0.007)$. Interestingly, prior studies showed that a higher pretreatment BMI indicates a longer failure-free survival in locoregionally advanced NPC and a longer overall survival in metastatic NPC. ${ }^{28,29}$ Higher BMI can be used as a protective factor for symptomatic VTE in NPC patients with PICC insertion. Due to the toxicity of radiotherapy and chemotherapy, loss of body weight is a continually observed problem among NPC patients. ${ }^{29,30}$ It was reported that the average loss of body weight was $7.85 \pm 4.32 \mathrm{~kg}$ from the first radiotherapy to 1 month posttreatment, and $50 \%$ patients suffered from $>10 \%$ weight loss at week 6 of radiotherapy plus chemotherapy using cisplatin. ${ }^{31,32}$ Lower BMI patients were more likely to develop malnutrition or cachexia, and previous studied have reported that cancer cachexia syndrome was in connection with thrombosis. ${ }^{10,33,34}$ In brief, malignant tumors interact with the hemostatic and coagulation system. Thromboembolism is resulted from prothrombotic factors generated by the malignancies, which can be exaggerated by cancer treatments and persists. Therefore, cancer cachexia patients are in hypercoagulable state. ${ }^{27,35} \mathrm{We}$ conclude that NPC patients may need more nutritional support and should maintain their body weight to prevent VTE during therapy.

Our findings regarding the relation between the risk factors and symptomatic PICC-VTE suggest that metastatic
NPC patients with VTE history, lower pretreatment BMI, or worse ECOG performance score require prophylactic use of anticoagulants.

Within our cohort, previously indicated risk factors for catheter-related VTE-like comorbidities, insertion arms, number of insertion attempts and lumens, and others were not predictive determinants. With a developed vascular access nursing team, we assumed that our standardized insertion technique may reduce the effects of certain technical variables that previously enhanced catheter-related VTE, allowing the presence of other more accurate predictive factors. In addition, patients enrolled in other studies were a combination of patients with various cancers rather than a single disease.

Furthermore, we did not find statistically predictive relationships between the coagulation function index, EBV, therapy regimens, and PICC-VTE in NPC patients. Whether biomarkers can predict the risk of VTE remains controversial. The results showed that the incidence of VTE has no connection with the amount of viral load or treatment modality.

We should emphasize some limitations in our study. First, this retrospective analysis was carried out in a single medical center, which may be susceptible to selection bias and incomplete clinical information like dominant hand or no-dominant hand. Second, the chemotherapy regimens for NPC patients in our study were not unified. Third, potential confounding variables such as PICC-related infections, or unidentified inherited VTE, and the incidence of asymptomatic PICC-VTE could be difficult to assess.

We recognize these limitations. However, we have important strengths, including a large sample size and a professional vascular care team that can not only insert PICC but also track outcomes over time. Moreover, we study only NPC patients, not a variety of different cancer patients, which can reduce the bias caused by difference with the malignant degree of differentiation.

\section{Conclusion}

PICC-related symptomatic VTE is a common complication in NPC patients receiving chemotherapy and/or radiotherapy. Those with VTE history and lower BMI and worse ECOG performance score metastatic NPC patients are more susceptible to symptomatic PICC-related thrombosis and thus may require prophylactic anticoagulation. More prospective clinical studies are needed to validate our conclusions.

\section{Data sharing statement}

The data sets used and/or analyzed during the current study are available from Yu-Ying Fan on reasonable request. 


\section{Acknowledgments}

We gratefully acknowledge the patients who participated in this study. This study was supported by grants from Medical Scientific Research Foundation of Guangdong Province, China (No A2014252, No A2014253).

\section{Disclosure}

The authors report no conflicts of interest in this work.

\section{References}

1. Ferlay J, Soerjomataram I, Dikshit R, et al. Cancer incidence and mortality worldwide: sources, methods and major patterns in GLOBOCAN 2012. Int J Cancer. 2015;136:E359-E386.

2. Torre LA, Bray F, Siegel RL, Ferlay J, Lortet-Tieulent J, Jemal A. Global cancer statistics, 2012. CA Cancer J Clin. 2015;65:87-108.

3. Orgel E, Ji L, Pastor W, Schore RJ. Infectious morbidity by catheter type in neutropenic children with cancer. Pediatr Infect Dis J. 2014; 33:263-266.

4. Lee AY, Levine MN, Butler G, et al. Incidence, risk factors, and outcomes of catheter-related thrombosis in adult patients with cancer. J Clin Oncol. 2006;24:1404-1408.

5. Chopra V, Ratz D, Kuhn L, Lopus T, Lee A, Krein S. Peripherally inserted central catheter-related deep vein thrombosis: contemporary patterns and predictors. J Thromb Haemost. 2014;12:847-854.

6. Bertoglio S, Faccini B, Lalli L, Cafiero F, Bruzzi P. Peripherally inserted central catheters (PICCs) in cancer patients under chemotherapy: a prospective study on the incidence of complications and overall failures. J Surg Oncol. 2016;113:708-714.

7. Chopra V, Kaatz S, Grant P, et al. Risk of venous thromboembolism following peripherally inserted central catheter exchange: an analysis of 23,000 hospitalized patients. Am J Med. 2018 Epub Feb 1.

8. Refaei M, Fernandes B, Brandwein J, Goodyear MD, Pokhrel A, Wu C. Incidence of catheter-related thrombosis in acute leukemia patients: a comparative, retrospective study of the safety of peripherally inserted vs. centrally inserted central venous catheters. Ann Hematol. 2016;95: 2057-2064.

9. Kucher N. Clinical practice. Deep-vein thrombosis of the upper extremities. $N$ Engl J Med. 2011;364:861-869.

10. Chopra V, Anand S, Hickner A, et al. Risk of venous thromboembolism associated with peripherally inserted central catheters: a systematic review and meta-analysis. Lancet. 2013;382:311-325.

11. Aw A, Carrier M, Koczerginski J, McDiarmid S, Tay J. Incidence and predictive factors of symptomatic thrombosis related to peripherally inserted central catheters in chemotherapy patients. Thromb Res. 2012;130:323-326.

12. Revel-Vilk S, Yacobovich J, Tamary H, et al. Risk factors for central venous catheter thrombotic complications in children and adolescents with cancer. Cancer. 2010;116:4197-4205.

13. Pabinger I, Thaler J, Ay C. Biomarkers for prediction of venous thromboembolism in cancer. Blood. 2013;122:2011-2018.

14. Chua M, Wee J, Hui EP, Chan A. Nasopharyngeal carcinoma. Lancet. 2016;387:1012-1024

15. Chang ET, Adami HO. The enigmatic epidemiology of nasopharyngeal carcinoma. Cancer Epidemiol Biomarkers Prev. 2006;15:1765-1777.

16. Khorana AA, Kuderer NM, Culakova E, Lyman GH, Francis CW. Development and validation of a predictive model for chemotherapyassociated thrombosis. Blood. 2008;111:4902-4907.

17. Verso M, Agnelli G. Venous thromboembolism associated with longterm use of central venous catheters in cancer patients. J Clin Oncol. 2003; 21:3665-3675.
18. Saber W, Moua T, Williams EC, et al. Risk factors for catheter-related thrombosis (CRT) in cancer patients: a patient-level data (IPD) metaanalysis of clinical trials and prospective studies. J Thromb Haemost. 2011;9:312-319.

19. Samama MM. An epidemiologic study of risk factors for deep vein thrombosis in medical outpatients: the Sirius study. Arch Intern Med. 2000;160:3415-3420.

20. Van Rooden CJ, Rosendaal FR, Meinders AE, Van Oostayen JA, Van Der Meer FJ, Huisman MV. The contribution of factor V Leiden and prothrombin G20210A mutation to the risk of central venous catheter-related thrombosis. Haematologica. 2004;89:201-206.

21. Extermann M, Boler I, Reich RR, et al. Predicting the risk of chemotherapy toxicity in older patients: the Chemotherapy Risk Assessment Scale for High-Age Patients (CRASH) score. Cancer. 2012;118: $3377-3386$

22. Hurria A, Togawa K, Mohile SG, et al. Predicting chemotherapy toxicity in older adults with cancer: a prospective multicenter study. $J$ Clin Oncol. 2011;29:3457-3465.

23. Freyer G, Geay JF, Touzet S, et al. Comprehensive geriatric assessment predicts tolerance to chemotherapy and survival in elderly patients with advanced ovarian carcinoma: a GINECO study. Ann Oncol. 2005;16: $1795-1800$

24. Khorana AA. Venous thromboembolism and prognosis in cancer. Thromb Res. 2010;125:490-493.

25. Paauw JD, Borders H, Ingalls N, et al. The incidence of PICC lineassociated thrombosis with and without the use of prophylactic anticoagulants. JPEN J Parenter Enteral Nutr. 2008;32:443-447.

26. Lyman GH. Venous thromboembolism in the patient with cancer: focus on burden of disease and benefits of thromboprophylaxis. Cancer. 2011;117:1334-1349.

27. Dammacco F, Vacca A, Procaccio P, Ria R, Marech I, Racanelli V. Cancer-related coagulopathy (Trousseau's syndrome): review of the literature and experience of a single center of internal medicine. Clin Exp Med. 2013;13:85-97.

28. Li W, Shen LJ, Chen T, et al. Overweight/obese status associates with favorable outcome in patients with metastatic nasopharyngeal carcinoma: a 10-year retrospective study. Chin J Cancer. 2016;35:75.

29. Huang PY, Wang CT, Cao KJ, et al. Pretreatment body mass index as an independent prognostic factor in patients with locoregionally advanced nasopharyngeal carcinoma treated with chemoradiotherapy: findings from a randomised trial. Eur J Cancer. 2013;49:1923-1931.

30. Zeng Q, Shen LJ, Guo X, Guo XM, Qian CN, Wu PH. Critical weight loss predicts poor prognosis in nasopharyngeal carcinoma. BMC Cancer. 2016;16:169.

31. Lin YH, Chang KP, Lin YS, Chang TS. Evaluation of effect of body mass index and weight loss on survival of patients with nasopharyngeal carcinoma treated with intensity-modulated radiation therapy. Radiat Oncol. 2015;10:136.

32. Chitapanarux I, Lorvidhaya V, Kamnerdsupaphon P, et al. Chemoradiation comparing cisplatin versus carboplatin in locally advanced nasopharyngeal cancer: randomised, non-inferiority, open trial. Eur J Cancer. 2007; 43:1399-1406.

33. Reddel CJ, Allen JD, Ehteda A, et al. Increased thrombin generation in a mouse model of cancer cachexia is partially interleukin- 6 dependent. J Thromb Haemost. 2017;15:477-486.

34. Falanga A, Marchetti M, Vignoli A. Coagulation and cancer: biological and clinical aspects. J Thromb Haemost. 2013;11:223-233.

35. Prandoni P, Lensing AW, Piccioli A, et al. Recurrent venous thromboembolism and bleeding complications during anticoagulant treatment in patients with cancer and venous thrombosis. Blood. 2002;100: 3484-3488. 
OncoTargets and Therapy

\section{Publish your work in this journal}

OncoTargets and Therapy is an international, peer-reviewed, open access journal focusing on the pathological basis of all cancers, potential targets for therapy and treatment protocols employed to improve the management of cancer patients. The journal also focuses on the impact of management programs and new therapeutic agents and protocols on

perspectives such as quality of life, adherence and satisfaction The manuscript management system is completely online and includes a very quick and fair peer-review system, which is all easy to use. Visit http://www.dovepress.com/testimonials.php to read real quotes from published authors.

Submit your manuscript here: http://www.dovepress.com/oncotargets-and-therapy-journal 